\title{
Corticosterone Predicts Foraging Behavior and Parental Care in Macaroni Penguins
}

\author{
Glenn T. Crossin, ${ }^{1,2, \star}$ Phil N. Trathan, ${ }^{3}$ Richard A. Phillips, ${ }^{3}$ Kristen B. Gorman, ${ }^{2}$ \\ Alistair Dawson, ${ }^{1}$ Kentaro Q. Sakamoto, ${ }^{4}$ and Tony D. Williams ${ }^{2}$
}

1. Centre for Ecology and Hydrology, Natural Environment Research Council, Bush Estate, Penicuik, Midlothian EH26 0QB, United Kingdom; 2. Biological Sciences Department, Simon Fraser University, Burnaby, British Columbia V5A 1S6, Canada; 3. British Antarctic Survey, Natural Environment Research Council, High Cross, Madingley Road, Cambridge CB3 0ET, United Kingdom; 4. Laboratory of Physiology, Graduate School of Veterinary Medicine, Hokkaido University, North 18 West 9, Kita-ku, Sapporo 060-0818, Japan

Submitted August 10, 2011; Accepted February 19, 2012; Electronically published May 24, 2012

Dryad data: http://dx.doi.org/10.5061/dryad.f385721n.

AвSTRACT: Corticosterone has received considerable attention as the principal hormonal mediator of allostasis or physiological stress in wild animals. More recently, it has also been implicated in the regulation of parental care in breeding birds, particularly with respect to individual variation in foraging behavior and provisioning effort. There is also evidence that prolactin can work either inversely or additively with corticosterone to achieve this. Here we test the hypothesis that endogenous corticosterone plays a key physiological role in the control of foraging behavior and parental care, using a combination of exogenous corticosterone treatment, time-depth telemetry, and physiological sampling of female macaroni penguins (Eudyptes chrysolophus) during the brood-guard period of chick rearing, while simultaneously monitoring patterns of prolactin secretion. Plasma corticosterone levels were significantly higher in females given exogenous implants relative to those receiving sham implants. Increased corticosterone levels were associated with significantly higher levels of foraging and diving activity and greater mass gain in implanted females. Elevated plasma corticosterone was also associated with an apparent fitness benefit in the form of increased chick mass. Plasma prolactin levels did not correlate with corticosterone levels at any time, nor was prolactin correlated with any measure of foraging behavior or parental care. Our results provide support for the corticosterone-adaptation hypothesis, which predicts that higher corticosterone levels support increased foraging activity and parental effort.

Keywords: allostasis, time-depth recorders, telemetry, parental care, reproductive investment, stress hormones.

\section{Introduction}

Corticosterone is the primary glucocorticoid produced by activation of the hypothalamic-pituitary-adrenal axis and

* Corresponding author. Present address: Department of Biology, Dalhousie University, Halifax, Nova Scotia B3H 4J1, Canada; e-mail: gtc@dal.ca.

Am. Nat. 2012. Vol. 180, pp. E31-E41. (C) 2012 by The University of Chicago. 0003-0147/2012/18001-53252\$15.00. All rights reserved.

DOI: $10.1086 / 666001$ facilitates the mobilization of energy and the acquisition of nutritional resources to allow behavioral responses to stressors (Sapolsky et al. 2000; Landys et al. 2006). Corticosterone has therefore received considerable attention as the principal hormonal mediator of allostasis or physiological stress in wild animals (Dallman et al. 1993). In particular, corticosterone is thought to play an adaptive role in the acquisition of energy and in overall energy balance, thus allowing individuals to respond to environmental perturbations or resource shortages during unpredictable, "emergency life-history stages" (Wingfield et al. 1998). However, as a key regulator of energy balance and locomotor activity, modulation of corticosterone may also allow an animal to adjust its "normal" day-to-day behavior, in specific environmental or physiological contexts, to meet the variable demands of predictable events such as reproduction (Holberton 1999; Ricklefs and Wikelski 2002; Breuner and Hahn 2003; McEwen and Wingfield 2003; Landys et al. 2006).

As an example of this potential metabolic, regulatory function, several recent studies have suggested that corticosterone might be positively correlated with parental care in breeding birds, particularly with respect to individual variation in foraging behavior and provisioning effort (Love et al. 2004; Angelier and Chastel 2009 and references therein). However, high levels of corticosterone can lead to the abandoning of offspring, and this presents a corticosterone-induced reproductive conflict (Love et al. 2004). Birds can resolve this conflict by upregulating endogenous corticosterone during the breeding season, from egg laying through to the end of chick rearing. By so doing, upregulation of endogenous corticosterone can support higher levels of foraging effort to match the energetic demands of growing chicks, but the relative sensitivity or 
threshold to acute stress also increases, thereby minimizing the potential for chick abandonment (Love et al. 2004; see also Pravosudov 2003; Angelier et al. 2007a, 2008, 2010; Bonier et al. 2011). It is thus predicted that endogenous corticosterone should be positively correlated with variation in current reproductive effort (e.g., measures of parental care and feeding rates) and ultimately fitness, an idea that forms the crux of the "corticosterone-adaptation" hypothesis (Bonier et al. 2009a). In essence, individuals that can more effectively or efficiently upregulate endogenous corticosterone to meet the metabolic demands of reproduction should have higher fitness. The corticosterone-fitness hypothesis (from which the corticosteroneadaptation hypothesis is derived; Bonier et al. 2009a) generally predicts a negative association between corticosterone and fitness such that high corticosterone reflects an individual or population in poor condition with low relative fitness (e.g., lower-quality parents with lower provisioning rates would have high corticosterone) or one that experiences poor environmental conditions. The distinction between the two hypotheses is subtle. Because corticosterone secretion and measures of individual fitness are not static traits of an individual, and because environmental conditions can be highly variable, the relationship between corticosterone and fitness can shift throughout an individual's lifetime and both hypotheses can predict negative relationships (see Bonier et al. 2009a). However, it is only the corticosterone-adaptation hypothesis that can predict positive associations between corticosterone and measures of fitness as discussed above.

Evidence is also emerging for a role of prolactin in the regulation of chick-brooding and chick-provisioning behavior (see review by Angelier and Chastel 2009), with a potential synergy between prolactin and corticosterone (Angelier and Chastel 2009). Miller et al. (2009) showed that variation in both baseline prolactin and baseline corticosterone levels in adult mourning doves (Zenaida macroura) was positively related to chick weight shortly after hatching, and they concluded that a synergism with prolactin minimizes the negative effects of corticosterone on reproduction while maintaining beneficial effects on increased foraging behavior and chick feeding. In blackbrowed albatrosses, high baseline corticosterone levels were positively correlated with the duration of incubation and brooding periods, while prolactin levels showed the opposite or inverse pattern, which is consistent with a corticosterone-prolactin stress response (Angelier et al. $2007 c$ ). However, few studies have sought to confirm the relationships among corticosterone, prolactin, parental care, and chick quality via experimental manipulation of corticosterone and direct assessment of foraging behavior in free-ranging birds. The studies described above suggest a complex, context-dependent relationship between cor- ticosterone and foraging effort during chick rearing, but additional experimental work is warranted. Experimental studies should include simultaneous examination of prolactin (Angelier and Chastel 2009) given its somewhat ambiguous role in parental care (Adkins-Regan 2005), even with respect to incubation behavior, which prolactin has long been held to directly regulate (Sockman et al. 2006 and references therein). Furthermore, experimental studies would allow a test of long-held ideas that corticosterone works in opposition to prolactin to favor allocation of resources to self-maintenance at the expense of allocation to current reproduction.

In this study, we test the hypothesis that corticosterone plays a key physiological role in the control of foraging behavior and parental care (Angelier et al. 2007b, 2009), and we simultaneously examine interrelationships with variation in plasma prolactin (Angelier and Chastel 2009). More specifically, we frame our study as a test of the corticosterone-adaptation hypothesis (Bonier et al. 2009a) using female macaroni penguins (Eudyptes chrysolophus) during the brood-guard stage of chick rearing as a model system. Macaroni penguins offer a particularly tractable model as females initiate multiple, consecutive foraging trips and are solely responsible for provisioning young chicks while males remain on the nest and sustain themselves through somatic energy reserves (Williams 1995; Barlow and Croxall 2002). Because females spend less time at the nest and are not principally responsible for chick brooding or guarding, this allows us to test the effect of corticosterone on foraging behavior and prolactin levels without the potentially confounding effect of long periods of food deprivation. Using exogenous corticosterone treatment at the onset of a foraging trip, we increased phenotypic variation in plasma corticosterone, and, based on the corticosterone-adaptation hypothesis, we predicted that relative to sham-implanted penguins, corticosteroneimplanted penguins (1) would increase their total foraging effort in terms of the time spent at sea, the time spent diving, the total number of dives initiated, and the total number of prey capture attempts exhibited while diving, (2) would experience a greater gain in body mass at the end of a foraging trip, and (3) would have significantly larger chicks toward the end of chick rearing. Finally, we predicted (4) an inverse relationship between prolactin and corticosterone, with corticosterone-implanted (henceforth "cort-implanted") females showing the lowest prolactin levels.

\section{Methods}

\section{Study Site and Field Sampling Protocol}

Fieldwork was conducted between January and February 2009 at a small breeding colony with approximately 500 
pairs of macaroni penguins on Fairy Point, Bird Island, South Georgia $\left(54^{\circ} 00^{\prime} \mathrm{S}, 38^{\circ} 02^{\prime} \mathrm{W}\right)$. Fairy Point is a good place to monitor the departures and returns of foraging penguins because the colony is fairly small and there is only one approach from the sea; penguins follow the same well-worn trail from the rocky shore up into the colony and use the same trail to return to sea. Research was conducted through permits issued by the British Antarctic Survey and conformed to guidelines established by the Canadian Committee on Animal Care (Simon Fraser University Animal Care Permit 897B-8).

We targeted female penguins at the beginning of the brood-guard stage of the reproductive season $\sim 5-7$ days after their eggs had hatched. Breeding pairs with young chicks were then identified on nests during the predawn hours, when most females are in the colony and preparing to leave for foraging trips (Barlow and Croxall 2002), and both partners were marked with a small dab of colored water-based paint on their chests. Macaroni penguins are sexually size dimorphic (Williams 1995), allowing for accurate identification of paired individuals; thus, females were dabbed yellow and males blue to allow easy sex identification. Study nests were selected randomly from the colony's perimeter to minimize colony disturbance. All birds were marked during a single morning, and we did not revisit the colony again until the next morning. The sex of captured females was subsequently confirmed by bill measurements (Williams and Croxall 1991) when females were captured and sampled at the beginning of foraging trips in the following days. On January 20, when mean age of chicks was approximately 15 days, all chicks were weighed $( \pm 10 \mathrm{~g})$ at their nests. This was just before the crèche period, when chicks leave nests and cluster in groups at around 15 days of age.

\section{Blood Sampling, Corticosterone Implantation, and Deployment of Time-Depth Recorders}

Marked females were captured at dawn as they made their way from the nests to the sea. On capture, 2.0-mL blood samples were taken immediately from brachial veins using heparinized syringes fitted with 25-gauge needles. Blood was transferred to heparinized 2.5-mL Eppendorf vials and centrifuged for $5 \mathrm{~min}$ at $10,000 \mathrm{~g}$, and plasma was then transferred to labeled $0.6-\mathrm{mL}$ vials and stored at $-20^{\circ} \mathrm{C}$ and then later at $-80^{\circ} \mathrm{C}$. We recorded the time $( \pm 1 \mathrm{~s})$ that it took to collect a blood sample from first approach to the end of blood collection. We recorded body mass $( \pm 10 \mathrm{~g})$ and bill length $( \pm 1 \mathrm{~mm})$ to confirm sex $(<22$ $\mathrm{mm}=$ female; Williams 1995). Before being released, each female received a subcutaneous passive integrated transponder tag bearing a unique identifying number and an 25-mm Silastic implant (internal diameter: $1.47 \mathrm{~mm}$, ex- ternal diameter: $1.96 \mathrm{~mm}$; Dow Corning, Midland, MI) filled with $15 \mathrm{mg}$ of crystallized corticosterone (C2505, Sigma Chemical, St. Louis, MO; $N=9$ ) or empty sham implants $(N=8)$. Implants were sealed at both ends, but one end was opened at the time of implantation to allow corticosterone release. Our goal was to temporarily increase plasma corticosterone titers above baseline within a physiologically realistic range (Holberton et al. 1996; Walker et al. 2005; Cockrem et al. 2006; Angelier et al. 2008; other seabird species: Chastel et al. 2005; Angelier et al. 2007a) and not to supraphysiological levels. The 15mg dose that we applied was approximately 50\% and $83 \%$ smaller than the doses used previously to manipulate corticosterone levels in seabirds (Angelier et al. 2009) and sea ducks (Criscuolo et al. 2005). Time-depth recorders (TDRs; Cefas Technology, G5 tags, Suffolk, United Kingdom) were deployed on penguins by attaching them with Tesa tape to feathers at the lower medial portion of the back. Individual TDRs weigh only $1 \mathrm{~g}$ in water, which represents $<0.001 \%$ of the mass of individual penguins. TDRs were programmed to record at 30-s intervals on the surface (depth $<5 \mathrm{~m}=$ surface travel) and at 0.5-s intervals once a dive was initiated (depth $>5 \mathrm{~m}$ ). The absolute accuracy of depth was $1 \mathrm{~m}$, and resolution was 0.5 m.

We sampled 17 female macaroni penguins on three occasions: January 4, 8, and 13, 2009. On each of these three release days, there were both cort- and sham-implanted individuals released (release 1: cort $=2$, sham $=2$; release 2: cort $=4$, sham $=4$; release 3: cort $=3$, sham $=2$ ). After females were sampled and released, we monitored penguin departures and returns continuously during daylight hours $(\sim 18 \mathrm{~h}$ per day). Penguins were then sampled on return to the colony after 1-3-day foraging trips at sea. These sampling dates spanned a 13-day period between January 4 and 16, 2009. When females returned from a foraging trip, they were recaptured en route to their nests, blood sampled, and measured as described above. TDRs were recovered at this time, and data were downloaded. No penguins were missed; we successfully recaptured all penguins en route to their nests after making single foraging trips. Foraging trip duration and activity patterns were subsequently extracted from the data. Departure and return dates were verified by visual observations of penguin movements.

\section{Hormonal Assays}

Corticosterone was determined by double antibody radioimmunoassay $\left({ }^{125}\right.$ I-RIA, MP Biomedicals, 07-120103) with modifications validated for several avian species (Washburn et al. 2002; Newman et al. 2008; Schmidt and Soma 2008). The assay detection limit was 3.13 pg corticosterone 
per tube (i.e., the lowest corticosterone standard, $12.5 \mathrm{ng}$ $\mathrm{mL}^{-1}$, using a $50-\mu \mathrm{L}$ assay volume). The low corticosterone control and a consistent native plasma sample were analyzed in each assay to determine an interassay coefficient of variation $(5.10 \%)$. Intra-assay coefficient of variation was $9.4 \%$, calculated from variation in a native plasma sample assayed 8 times within a single assay. Serially diluted native plasma samples were parallel to the corticosterone standard curve (coefficients of variation for final concentrations were $12.24 \%, N=3$ ) following methods outlined in Plikaytis et al. (1994). All samples were assayed in duplicate.

Prolactin was assayed in duplicate $20-\mu \mathrm{L}$ plasma samples in a recombinant-derived starling prolactin assay (Bentley et al. 1997). The sensitivity of the assay was $1.0 \mathrm{ng} \mathrm{mL}^{-1}$, and $50 \%$ displacement was obtained with $12.1 \mathrm{ng} \mathrm{mL}^{-1}$. All samples were measured in a single assay, and the intraassay coefficient of variation (CV) was $6.5 \%$. Intra-assay CVs were calculated using eight duplicate aliquots from a pool of plasma (not standards) in each case. For some samples, a second assay at $10-\mu \mathrm{L}$ volume was measured to ensure sample parallelism with the standard curve.

\section{TDR Data Analysis}

Dive-depth data were analyzed using the program-package Ethographer (Sakamoto et al. 2009) for Igor Pro (WaveMetrics, Lake Oswego, OR). For each individual, the total number of dives was estimated, and for each dive, the depths, durations, and number of prey capture attempts (or "wiggles"; Takahashi et al. 2004) were determined. Wiggles were defined as dive events when birds changed their swimming direction rapidly from descending to ascending to descending (Takahashi et al. 2004). This has been previously shown as a reliable proxy for prey capture during foraging events and correlates positively with bill openings and closings (Ropert-Coudert et al. 2001; Simeone and Wilson 2003; Takahashi et al. 2004; Bost et al. 2007). Because of surface noise, we used a conservative approach and analyzed dives $>10 \mathrm{~m}$ in depth, which is the suggested depth threshold for foraging dives of macaroni penguins at Bird Island, South Georgia (Green et al. 2005).

\section{Statistical Analyses}

Analyses were run with the JMP 7.0 or SAS 9.0 software package. All variables were tested for normal distribution via plots of residuals against predicted values followed by Shapiro-Wilk tests for normality $(P<.05)$. After $\log _{10}$ transformations, all variables approached normality. A repeated-measures mixed linear model (SAS PROC MIXED) was used to compare temporal, intraindividual changes in corticosterone and prolactin concentrations from samples collected before and after foraging trips. Tukey-Kramer post hoc tests were run to identify significant contrasts between stages. Multivariate analysis of covariance (MANCOVA) was used to explore treatmentrelated differences (i.e., cort- vs. sham-implanted) with respect to multiple foraging behaviors (number of dives initiated, maximum depth of dives, number of discrete foraging events during dives [or "wiggles"]; Takahashi et al. 2004), while controlling for variation in the time that penguins spent away from the colony during foraging trips (treatment, total time away, and interaction term). If the MANCOVA model was significant, separate univariate ANCOVA tests were run to identify the variables driving the significant MANCOVA. Pearson's correlations were used to explore relationships among dive behaviors derived from TDRs (e.g., time away from colony, total dive number, dive foraging events) and penguin attributes (total mass gained while foraging and the mass of chicks measured once before crèche on January 27, 2009, 14-23 days after implantation). Finally, linear regression models were used to explore the influence of postforaging corticosterone levels (continuous independent variable) against various dependent variables (e.g., total dive duration, number of dives, number of attempted prey captures, female mass gain, and chick mass). Because these dependent variables were all correlated with time that penguins spent away from the colony, we used regression analysis to generate time-corrected residuals. These were then regressed against postforaging corticosterone to explore the influence of this variable on foraging behavior. All values presented in figures are individual data or untransformed least squares means \pm SEM.

\section{Results}

The mean times to collect pre- and postforaging blood samples were $157 \pm 32.2 \mathrm{~s}$ (mean $\pm \mathrm{SD}$; range $=$ $100-220 \mathrm{~s}$ ) and $173 \pm 41.1 \mathrm{~s}$ (range $=100-250 \mathrm{~s}$ ), respectively. There was no significant relationship between these times with either pre- or postforaging prolactin and corticosterone concentrations (all $P>.288$ ).

There was a significant pre- to postforaging trip difference in corticosterone levels between treatment groups, indicating that individual corticosterone profiles increased significantly in cort-implanted females but not in shamimplanted females (repeated-measures ANOVA, full model $F_{1,15}=7.13, P=.018$; fig. $\left.1 A\right)$. Note that the two penguins with postimplantation corticosterone levels in excess of $130 \mathrm{ng} \mathrm{mL} \mathrm{mL}^{-1}$ did not initiate foraging trips (confirmed by visual observations and telemetry data) but nevertheless left the colony for a short period of time $(<12 \mathrm{~h}$ spent on the rocks at colony entrance, not foraging at sea). They were thus excluded from subsequent analyses of foraging 

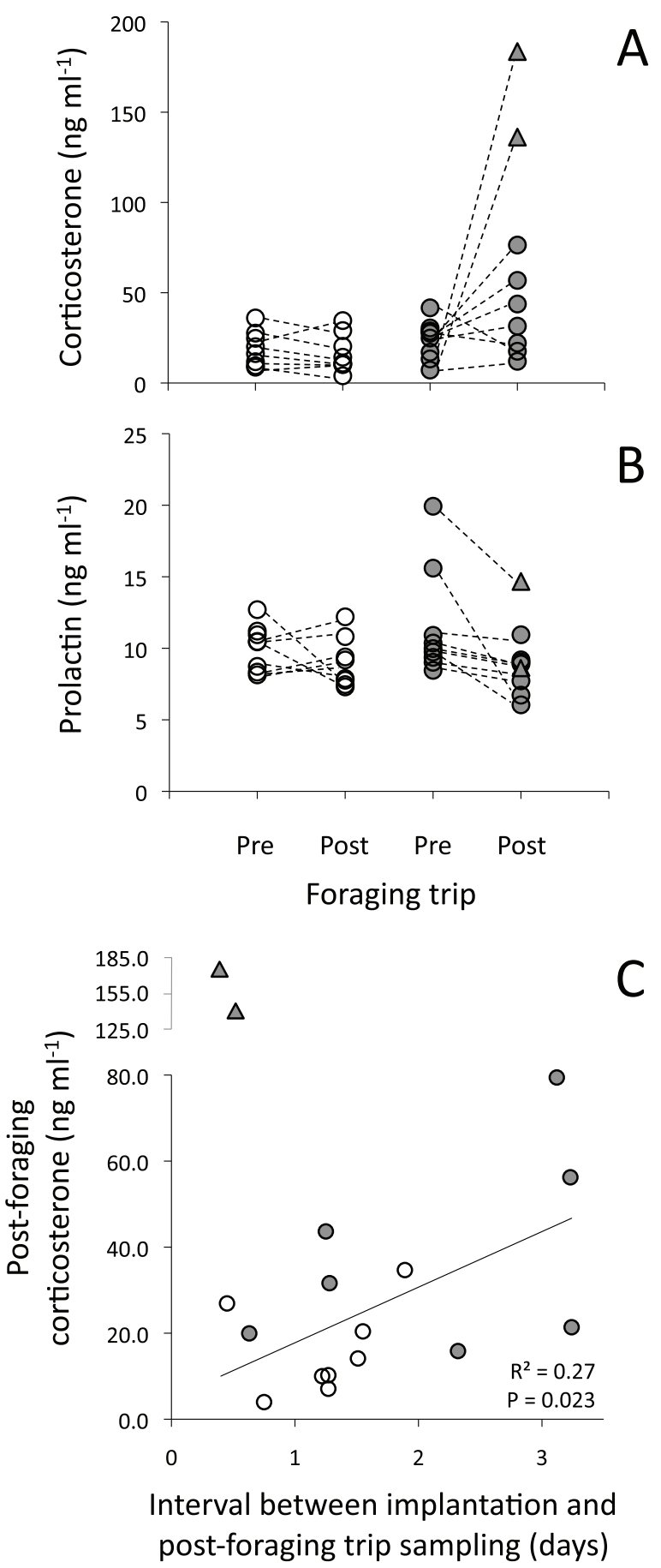

Figure 1: Change of corticosterone concentrations $(A)$ in individual female macaroni penguins (Eudyptes chrysolophus) that were given corticosterone implants (gray circles) or sham implants (white circles). Change in prolactin concentrations $(B)$ are also presented and grouped by treatment group. Note differences in $Y$-axis scale. The "pre" samples reflect baseline corticosterone before implantation and marine foraging. The "post" samples reflect postimplantation levels measured directly after 1-3-day foraging trips. All females were sampled during the brood-guard stage of the breeding season, during behavior. With regard to prolactin, there was a significant decrease in pre- to postforaging levels, but no difference between treatment groups was detected (full model $F_{1,15}=0.63, P=.441$; fig. $1 B$ ).

Because we do not know with certainty the rate at which corticosterone is released from Silastic capsules, we regressed postforaging concentrations against time away from the colony in order to determine whether penguins that return to the colony quickly have the highest corticosterone levels (i.e., is there an inverse relationship between postforaging corticosterone and time away from the colony?). When all 17 penguins were included in the analysis, there was no significant relationship between postforaging corticosterone and time spent away from the colony $\left(F_{1,15}=0.517, P=.483\right)$. When the two penguins with supraphysiological levels were excluded, a significant, positive linear relationship exists $\left(F_{1,13}=7.749, P=.016\right)$, meaning that corticosterone levels were highest in the penguins making the longest trips (fig. 1C).

There were significant correlations among all dive behaviors and with dive behavior and female mass gain and chick mass (Pearson's correlations: total time away from colony vs. total number of foraging dives, $r=0.83$, $N=15, P<.001$; total number of foraging dives vs. total number of foraging events or wiggles, $r=0.98, N=15$, $P<.001$; number of wiggles vs. female mass gain, $r=$ $0.82, N=15, P<.001$; female mass gain vs. chick mass, $r=0.77, N=17, P<.001$; see fig. 2 ).

Because individuals varied in the amount of time spent away from the colony during foraging trips, all dive/foraging variables were regressed against time away to generate "time-corrected" residuals. These were then regressed against postforaging corticosterone levels to explore the possible influence of corticosterone on behavior. In support of our first prediction, significant, positive linear relationships were found between postforaging corticosterone levels and foraging behavior: the total cumulative depth of all dives initiated $\left(F_{1,13}=8.756, P=.011\right.$; fig. $3 A)$ with the amount of time spent diving $\left(F_{1,13}=8.329\right.$, $P=.013$; fig. $3 B$ ) and with the number of dives initiated $\left(F_{1,13}=5.137, P=.041\right.$; fig. $\left.3 C\right)$. Postforaging corticosterone levels were not significantly related to the number of attempted prey captures (i.e., wiggles; $F_{1,13}=2.172$, $P=.163$; fig. $3 D$ ). In support of our second and third

which short foraging trips are made at sea to provision growing chicks while the male partner remains on the nest. The relationship between postforaging corticosterone levels and the time interval between cort implantation and postforaging sampling is also presented $(C)$. Note that the two penguins with postforaging corticosterone levels $>130 \mathrm{ng} \mathrm{mL}^{-1}$ (triangles) did not initiate foraging trips and were thus excluded from the regression, as well as from subsequent analyses of dive/foraging behavior. 


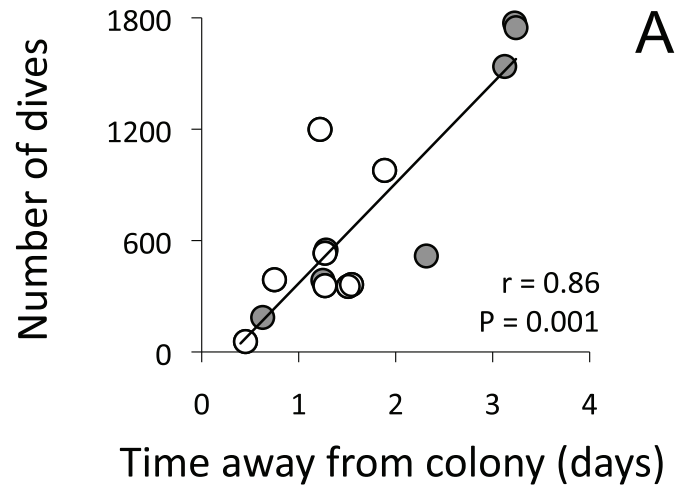

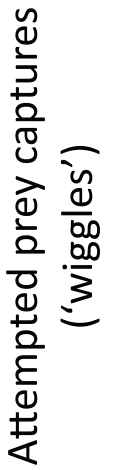
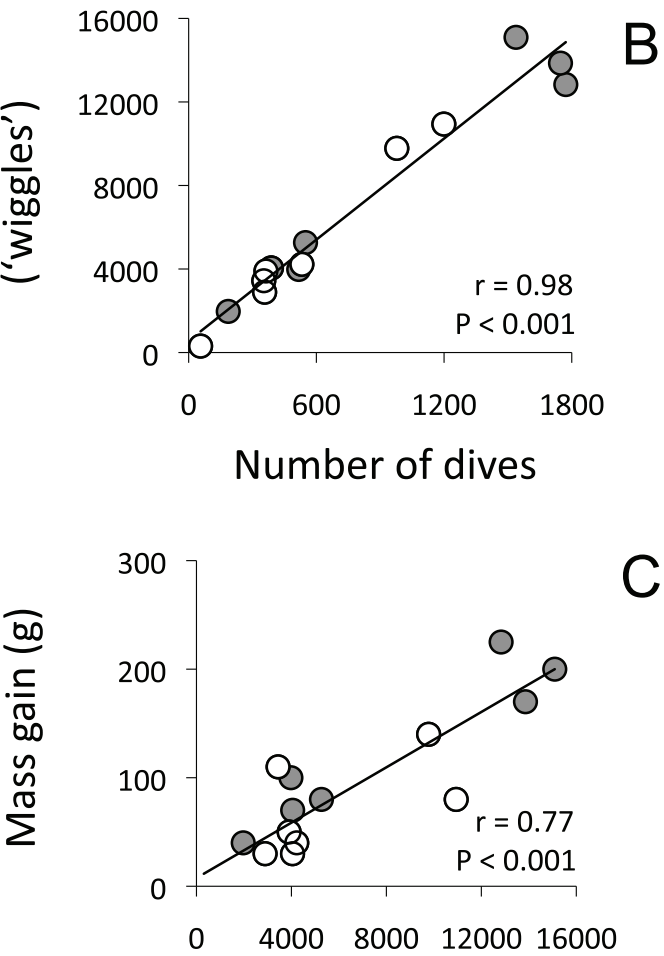

Attempted prey captures ('wiggles')

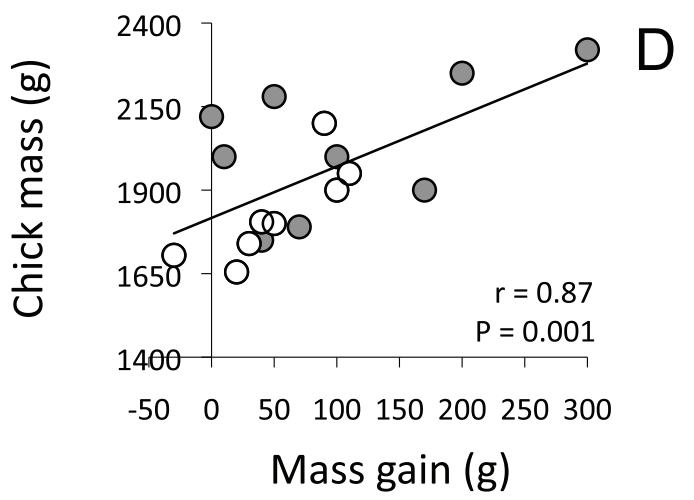

predictions, postforaging corticosterone levels were also significantly related to female mass gain $\left(F_{1,13}=10.040\right.$, $P=.007)$ and to chick mass $\left(F_{1,13}=15.752, P=.002\right.$; fig. 4). In contrast, there were no significant linear relationships between preforaging corticosterone levels and any of the dive/foraging variables (all $P>.10$ ), nor were there significant linear relationships with pre- or postforaging prolactin levels (all $P>.25$ ).

With the exception of a significant correlation between pre- and postforaging corticosterone $(r=0.59, N=17$, $P=.028)$, there were no significant correlations between corticosterone and prolactin at any time $(N=17$, all $P>$.20). The lack of significant relationship between corticosterone and prolactin provides no support for our fourth prediction.

Because total time away from the colony was correlated with all foraging variables (all $P<.001$ ), we controlled for variation in time away when exploring differences in dive behavior by treatment group. A MANCOVA model was thus run to explore foraging trip attributes (e.g., total dive duration, total number of dives initiated, total cumulative dive depth, and total number of prey capture attempts or wiggles). In support of our first prediction, the model revealed a significant effect of treatment and time away from colony (whole model, Wilks's $\lambda$ approximate $F_{8,18}=$ 6.987, $P<.001$; treatment effect, exact $F_{4,9}=7.421, P=$ .006; time away from colony effect, exact $F_{4,9}=4.236$, $P=.034)$. The interaction term (treatment $\times$ time away from colony) was not significant $(P=.597)$, so it was removed from the model to increase statistical power. Centroid plots suggested that cort-implanted females initiated more dives than sham-implanted females. Subsequent ANCOVA models using treatment as the main effect and time away from colony as the covariate confirmed this and lend support to our first predictions (total dive number: whole model, $\left.F_{2,12}=446.3, P<.001\right)$. Furthermore, there were significant treatment differences in female mass gain (whole model, $F_{2,12}=29.9, P<.001$ ) and chick mass (whole model, $F_{2,12}=11.8, P=.001$; fig. $5 B-5 D$ ), which supports our second and third predictions. There were no other significant treatment effects on behavior (total cumulative dive depth, total duration of dive bottoms, total prey capture/wiggle events; all $P>.40$ ).

Preforaging corticosterone levels were similar between

Figure 2: Relationships between dive behavior $(A, B)$, mass gain $(C)$, and a measure of parental care (chick growth; $D$ ) in female macaroni penguins (Eudyptes chrysolophus). Statistics are Pearson's correlations. Gray circles indicate females that were implanted with corticosterone before leaving for sea, and white circles indicate sham-implanted females. Linear fits are used to illustrate the positive relationships. 


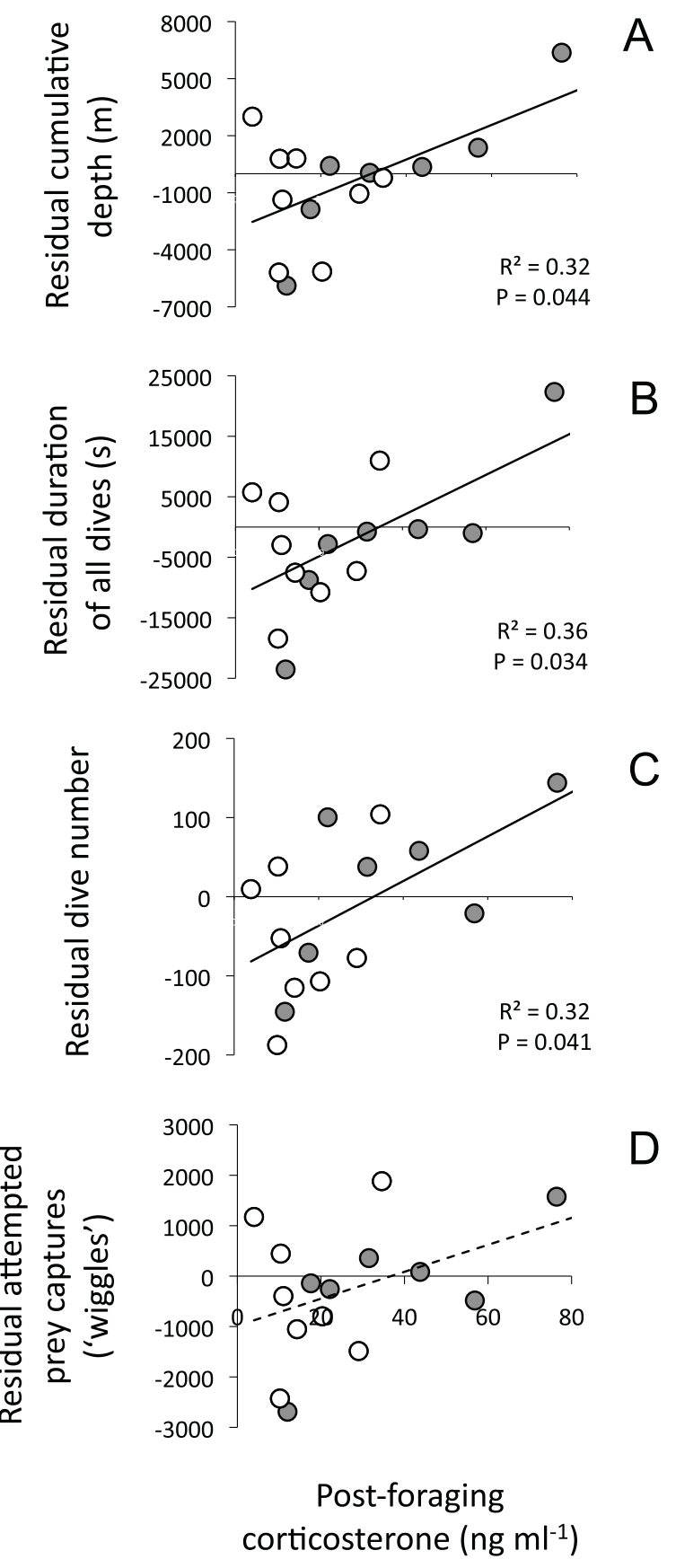

Figure 3: Linear relationships between baseline corticosterone and aspects of foraging behavior in female macaroni penguins (Eudyptes chrysolophus): the cumulative depth of dives $(A)$, the total time spent diving while away from the breeding colony $(B)$, the total number of dives initiated $(C)$, and the cumulative number of attempted prey captures or "wiggles" made during dives $(D)$. Gray circles indicate females that were implanted with corticosterone before leaving for sea, and white circles indicate sham-implanted females. Lines are best linear fits. The dashed line indicates a fit that was not statistically significant.
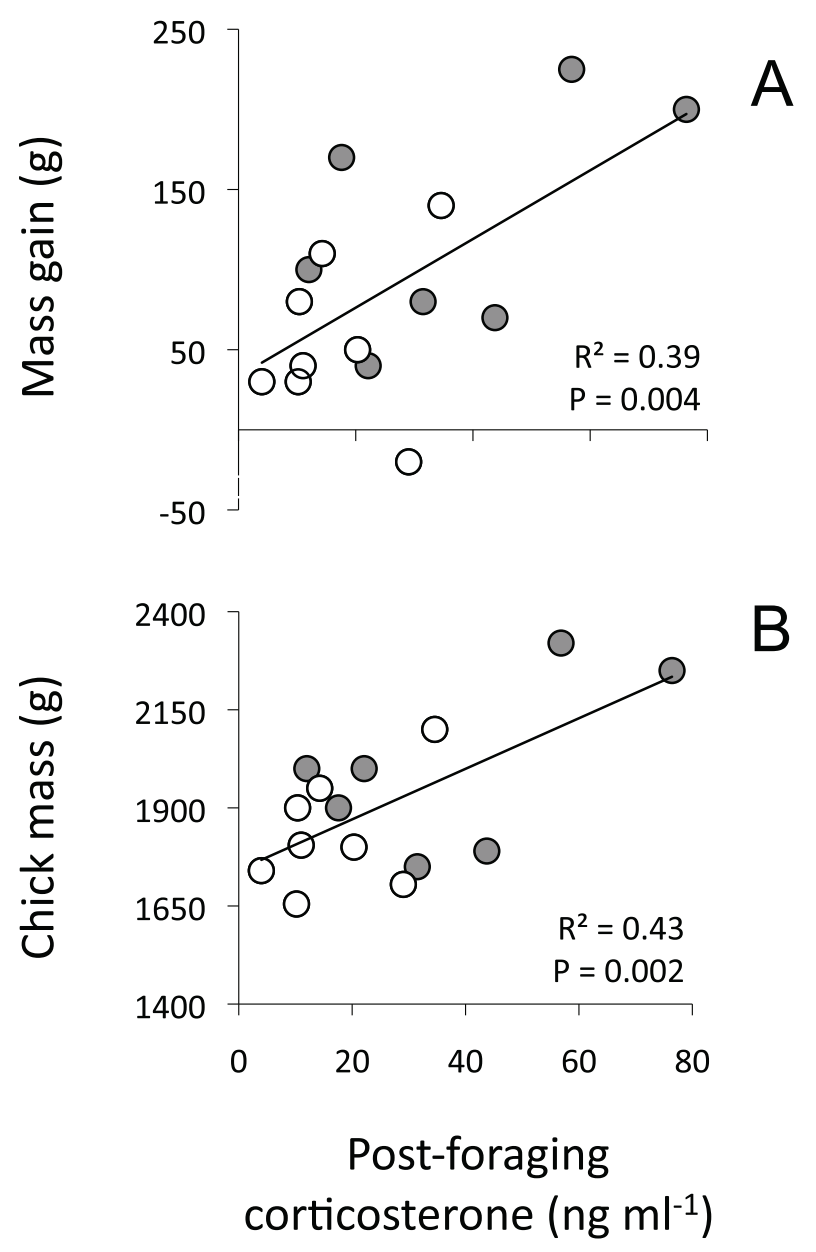

Figure 4: Linear relationships between postforaging baseline corticosterone levels in female macaroni penguins (Eudyptes chrysolophus) and adult mass gain $(A)$ and mass of chicks at 15 days of age $(B)$. Gray circles indicate females that were implanted with corticosterone before leaving for sea, and white circles indicate sham-implanted females. Lines are best linear fits.

treatment groups (ANCOVA, $F_{2,12}=1.25, P=.332$ ), but postforaging levels were significantly higher in cortimplanted females $\left(F_{2,12}=7.62, P=.007\right.$; fig. $\left.5 A\right)$. No treatment-related differences in prolactin levels were detected (preforaging, $F_{2,12}=0.28, P=.762$ ).

\section{Discussion}

In this study we used experimental manipulations of plasma corticosterone in female macaroni penguins and combined this with time-depth telemetry and assessment of chick growth to test one of the key predictions of the corticosterone-adaptation hypothesis (Bonier et al. 2009a): 

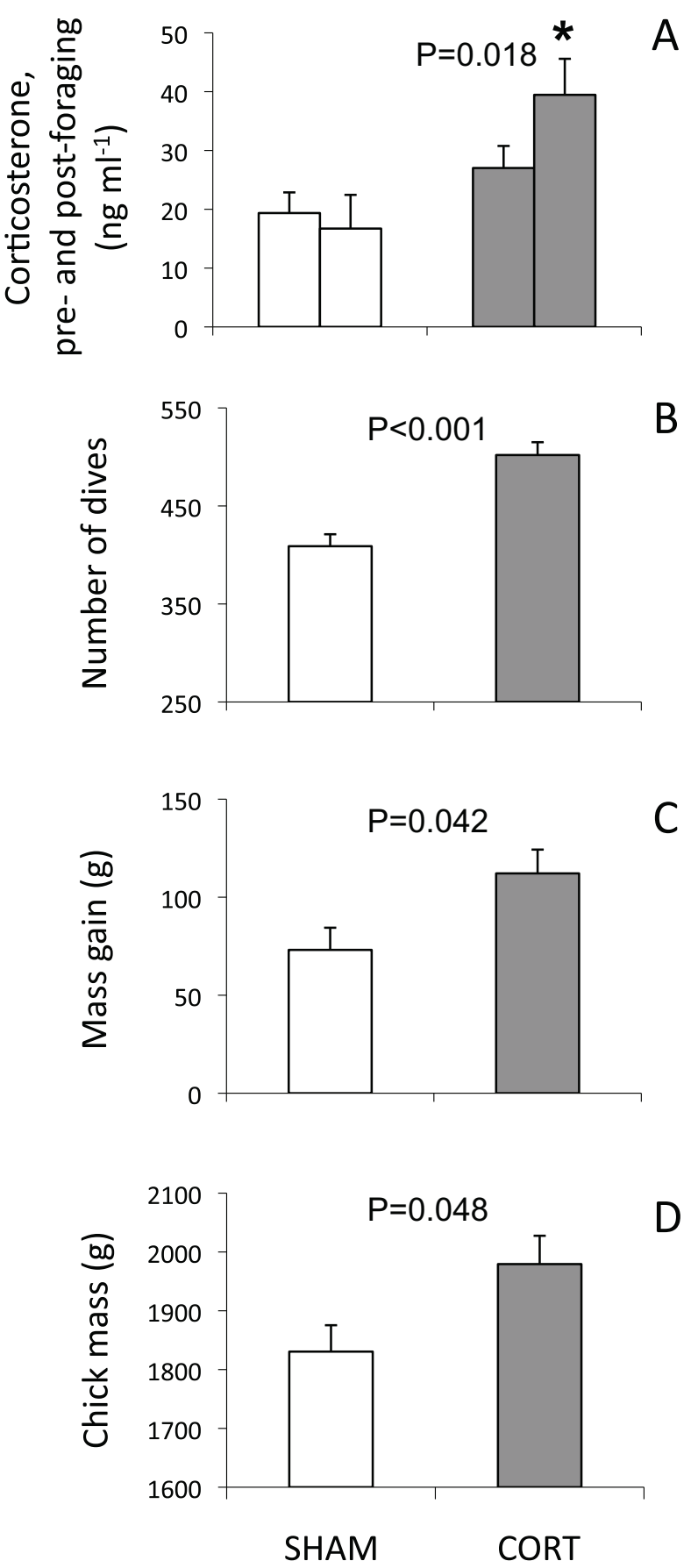

Figure 5: Differences between treatment groups in mean corticosterone concentrations in female macaroni penguins (Eudyptes chrysolophus) before and after a foraging trip $(A)$, in the total number of dives initiated while foraging $(B)$, in the mass gained during foraging $(C)$, and in the mass of chicks at 15 days of age $(D)$. Bars reflect least squares means \pm SEM, which account for individual variation in total dive duration. Full ANCOVA model $P$ values are presented in "Methods," while $P$ values for treatmentrelated effects are presented in the panels above. that plasma corticosterone should be positively correlated with current reproduction given corticosterone's key metabolic regulatory function and its role in supporting elevated levels of foraging and chick provisioning. Our data strongly support this hypothesis. Specifically, we found that individual variation in plasma corticosterone levels measured at the end of foraging trips correlated significantly with diving behavior such that high levels led to increases in foraging activity (fig. 3), an effect very strongly related to exogenous corticosterone treatment. Increased foraging effort by cort-implanted females then resulted in an increase in body mass and a greater delivery of food resources to chicks, which, at approximately 15 days of age, were significantly heavier than the chicks of lighter sham-treated females. These results are striking because they provide a corticosterone-dependent mechanism that supports the central premise of the corticosterone-adaptation hypothesis (Bonier et al. 2009a), which states that environmental and reproductive challenges can promote the acquisition and allocation of resources to reproduction, thereby leading to a direct fitness-related effect.

A concern with the use of corticosterone implants is that they can elevate plasma corticosterone from "baseline" to acute or maximum "stress-induced" levels, or to even higher pharmacological levels, which would then be predicted to have negative effects on current reproduction (e.g., Silverin 1986; Breuner et al. 2008). Our aim was to raise plasma levels but without causing an activation of type 2 glucocorticoid receptors, which typically occurs during allostatic overload and acute stress events with consequences for behavior and physiology. We thus used a relatively low dose of corticosterone (half of that used by Criscuolo et al. 2005) in an effort to minimize the potential for negative effects via type 2 receptor binding. The result was an increase in the phenotypic range of baseline corticosterone (and thus type 1 but not type 2 receptor binding) without creating the bimodal distribution of baseline versus stress-induced levels that is often observed in studies using standardized handling stressor protocols or exogenous implants (corticosterone was normally distributed across all cort- and sham-implanted penguins; Shapiro-Wilk goodness of fit, $W=0.9800, P=.958$ ). Our implants resulted in plasma corticosterone levels that were on average twofold higher in cort- versus shamimplanted penguins, and in only two cort-implanted individuals did levels exceed $100 \mathrm{ng} \mathrm{mL}^{-1}$. We were therefore successful in increasing the range of baseline variation without exerting any discernible negative effects on current reproduction: none of the penguins in our study abandoned their chicks, and mean chick mass was higher for cort-implanted mothers, even the two with highly elevated corticosterone. Based on these results, we contend that we did not induce an acute stress response in our penguins 
or induce an "emergency life-history stage" (sensu Wingfield et al. 1998) where resources are diverted from current reproduction to survival (Breuner et al. 2008). Alternatively, we show that exogenous upregulation of baseline corticosterone promotes homeostatic regulation to support a higher allocation of resources to reproduction, thus supporting the corticosterone-adaptation hypothesis. In contrast, both the corticosterone-fitness hypothesis (Bonier et al. 2009a) and the concept of an acute stress-induced corticosterone response predict negative relationships between plasma corticosterone, parental quality, and current reproduction. Our data do not support either of these concepts or interpretations and instead are more closely aligned with several recent studies that show positive relationships between elevated corticosterone, increased locomotor activity, and foraging behavior (Astheimer et al. 1992; Pravosudov 2003; Bonier et al. 2011), as well as increased parental quality and parental care (Love et al. 2004; Angelier et al. 2007a, 2008; Bonier et al. 2009b, 2011).

When examined from these perspectives, the experimentally elevated corticosterone levels that we created in the cort-implanted penguins have a broader significance. The functional significance of increased corticosterone levels lies in matching circulating levels to specific life-history and breeding environment characteristics (Williams 2008). In general, corticosterone levels in females tend to be higher in species in which males provide the bulk of parental care in terms of incubation and brooding activity while females forage, and levels also tend to be higher in species breeding at higher latitudes (Bókony et al. 2008), two scenarios that characterize the breeding system of female macaroni penguins (Williams 1995) and lay the foundations for the "preparative hypothesis" (sensu Romero 2002). Our results provide experimental support for this hypothesis, while fitting within the greater framework of the corticosterone-adaptation hypothesis. The general increase in plasma corticosterone levels that we measured in cort-implanted females were within a physiologically realistic range $\left(17-76 \mathrm{ng} \mathrm{mL}^{-1}\right)$. This had a permissive or "preparative" effect on individual foraging effort (sensu Romero 2002) that resulted in greater foraging effort in terms of the number of dives initiated while away at sea, on the duration of diving activity, and on the total cumulative depth of all dives (all variables were corrected to account for variation in time away from the colony). Ultimately, this preparative effect of corticosterone resulted in the delivery of more food to chicks (fig. 5D), which constitutes a potential direct fitness benefit.

Recent studies that have suggested that prolactin might work synergistically with corticosterone to regulate aspects of chick rearing (reviewed by Angelier and Chastel 2009). However, in our study plasma prolactin levels were not significantly correlated with variation in diving and foraging behavior and showed no relationship with chick growth (e.g., no support for prediction 4). In some species (including altricial birds), prolactin can be elevated throughout incubation and chick rearing, which has been interpreted as physiological involvement in the control of not only incubation but also chick brooding and provisioning (Angelier and Chastel 2009). For example, in some seabird species, prolonged foraging trips during incubation and chick rearing are often accompanied by elevated prolactin levels. In a study of king penguins (Aptenodytes patagonicus), prolactin levels were maintained at high values $\left(>40 \mathrm{ng} \mathrm{mL} \mathrm{m}^{-1}\right.$ ) through incubation and the first chickfeeding period (Garcia et al. 1996). Then, after a 5-month period at sea, parents returned for a second chick-feeding period with prolactin levels that were as high as when they departed. Similarly, in other penguins (Lormee et al. 1999; Vleck et al. 2000) and albatrosses (Hector and Goldsmith 1985), individuals returning to breeding colonies after foraging trips show little variation in prolactin levels relative to their incubating partner, and levels do not increase once incubation duties resume. Similar data from the broodguarding period are not readily available, but our data suggest that variation in prolactin is not directly involved in the regulation of foraging behavior of macaroni penguins or in the level of provisioning. We did observe small but significant reductions in baseline prolactin levels from pre- to postforaging, but this change was not influenced by corticosterone treatment. Furthermore, neither pre- nor postforaging prolactin levels correlated with any aspect of diving behavior or with chick growth. This suggests that prolactin secretion might be endogenously timed with little bearing on foraging behavior. That we have not observed a role of prolactin at this stage of the breeding season is perhaps not surprising given that, despite years of study, its involvement in the control of even incubation behavior, let alone parental care more generally, is not well understood (see Sockman et al. 2006 and references therein).

In summary, we found that experimental manipulation of plasma corticosterone had a positive effect on foraging behavior, which resulted in direct increases in chick growth even in females that were pushed toward very high levels and had temporarily suspended foraging activity. Collectively, these observations may foretell an adaptive tactic that maintains high rates of provisioning when investment in current reproduction is high, and when only one parent is provisioning in what can be highly unpredictable polar environments (e.g., the Southern Ocean), where multiple stressors occur (e.g., predation [Walker et al. 1998], extreme weather [Romero et al. 2000], and high thermoregulatory costs [Romero 2002]). These data are consistent with the idea that continuous, phenotypic variation in plasma corticosterone, as a homeostatic, metabolic regu- 
lator, is positively correlated with individual variation ir. $\rightarrow$ current reproductive effort, as predicted by the corticosterone-adaptation hypothesis, which presumably exerts a positive effect on fitness.
Bentley, G. E., A. R. Goldsmith, A. Dawson, L. M. Glennie, R. T. Talbot, and P. J. Sharp. 1997. Photorefractoriness in European starlings (Sturnus vulgaris) is not dependent upon the long-dayinduced rise in plasma thyroxine. General and Comparative Endocrinology 107:428-438.

$\rightarrow$ Bókony, V., Á. Z. Lendavi, A. Liker, F. Angelier, J. C. Wingfield, and O. Chastel. 2009. Stress response and the value of reproduction: are birds prudent parents? American Naturalist 173:589-598.

\section{Acknowledgments}

We extend thanks to S. Adlard and F. Le Bouard for field assistance and to P. Sharp for assistance with the prolactirassay. E. Chin, O. Love, K. Salvante, and members of the Soma Lab at the University of British Columbia provided critical advice regarding the corticosterone radioimmu. noassay. This work was supported by the British Antarctic Survey's Antarctic Funding Initiative Collaborative Gearing Scheme awarded to A.D., P.N.T., and R.A.P., by a Natural Sciences and Engineering Research Council of Canada (NSERC) Postdoctoral Fellowship and E-Bird funding to G.T.C., and by an NSERC Discovery Grant to T.D.W.

\section{Literature Cited}

Adkins-Regan, E. 2005. Hormones and animal social behavior. Princeton University Press, Princeton, NJ.

$\rightarrow$ Angelier, F., C. A. Bost, M. Giraudeau, G. Bouteloup, S. Dano, ana O. Chastel. 2008. Corticosterone and foraging behavior in a diving seabird: the Adélie penguin (Pygoscelis adeliae). General and Comparative Endocrinology 156:134-144.

$\rightarrow$ Angelier, F., and O. Chastel. 2009. Stress, prolactin and parentaı investment in birds: a review. General and Comparative Endocrinology 163:142-148.

$\rightarrow$ Angelier, F., C. Clement-Chastel, G. W. Gabrielsen, and O. Chastel. 2007a. Corticosterone and time-activity budget: an experimen with black-legged kittiwakes. Hormones and Behavior 52:482-491.

$\rightarrow$ Angelier, F., M. Giraudeau, C.-A. Bost, F. Le Bouard, and O. Chastel. 2009. Are stress hormone levels a good proxy of foraging success? an experiment with king penguins, Aptenodytes patagonicus. Journal of Experimental Biology 212:2824-2829.

$\rightarrow$ Angelier, F., S. A. Shaffer, H. Weimerskirch, C. Trouve, and O. Chastel. $2007 \mathrm{~b}$. Corticosterone and foraging behavior in a pelagic seabird. Physiological and Biochemical Zoology 80:283-292.

$\rightarrow$ Angelier, F., H. Weimerskirch, S. Dano, and O. Chastel. 2007c. Age, experience and reproductive performance in a long-lived bird: a hormonal perspective. Behavioral Ecology and Sociobiology 61: 611-621.

$\rightarrow$ Angelier, F., J. C. Wingfield, H. Weimerskirch, and O. Chastel. 2010. Hormonal correlates of individual quality in a long-lived bird: a test of the "corticosterone-fitness hypothesis." Biology Letters 6. 846-849.

$\rightarrow$ Astheimer, L. B., W. A. Buttemer, and J. C. Wingfield. 1992. Interactions of corticosterone with feeding, activity and metabolism ir_ $\rightarrow$ passerine birds. Ornis Scandinavica 23:355-365.

$\rightarrow$ Barlow, K. E., and J. P. Croxall. 2002. Seasonal and interannual variation in foraging range and habitat of macaroni penguins Eudypte. chrysolophus at South Georgia. Marine Ecology Progress Series 232: 291-304. $\rightarrow$ Bonier, F., P. R. Martin, I. T. Moore, and J. C. Wingfield. 2009a. Do baseline glucocorticoids predict fitness? Trends in Ecology \& Evolution 24:634-642.

Bonier, F., I. T. Moore, P. R. Martin, and R. J. Robertson. $2009 b$. The relationship between fitness and baseline glucocorticoids in a passerine bird. General and Comparative Endocrinology 163:208213.

$\rightarrow$ Bonier, F., I. T. Moore, and R. J. Robertson. 2011. The stress of parenthood? increased glucocorticoids in birds with experimentally enlarged broods. Biology Letters 7:944-946.

Bost, C. A., Y. Handrich, P.J. Butler, A. Fahlman, L.G. Halsey, A.J. Woakes, and Y. Ropert-Coudert. 2007. Changes in dive profiles as an indicator of feeding success in king and Adélie penguins. Deep Sea Research II 54:248-255.

$\rightarrow$ Breuner, C. W., and T. P. Hahn. 2003. Integrating stress physiology, environmental change, and behavior in free-living sparrows. Hormones and Behaviour 43:115-123.

$\rightarrow$ Breuner, C. W., S. H. Patterson, and T. P. Hahn. 2008. In search of relationships between the acute adrenocortical response and fitness. General and Comparative Endocrinology 157:288-295.

$\rightarrow$ Chastel, O., A. Lacroix, H. Weimerskirch, and G. W. Gabrielsen. 2005. Modulation of prolactin but not corticosterone responses to stress in relation to parental effort in a long-lived bird. Hormones and Behavior 47:459-466.

$\rightarrow$ Cockrem, J. F., M. A. Potter, and E. J. Candy. 2006. Corticosterone in relation to body mass in Adélie penguins (Pygoscelis adeliae) affected by unusual sea ice conditions at Ross Island, Antarctica. General and Comparative Endocrinology 149:244-252.

Criscuolo, F., O. Chastel, F. Bertile, G. W. Gabrielsen, Y. Le Maho, and T. Raclot. 2005. Corticosterone alone does not trigger a short term behavioural shift in incubating female common eiders Somateria mollissima, but does modify long term reproductive success. Journal of Avian Biology 36:306-312.

$\rightarrow$ Dallman, M. F., A. M. Strack, S. F. Akana, M. J. Bradbury, E. S. Hanson, K. A. Scribner, and M. Smith. 1993. Feast and famine: critical role of glucocorticoids with insulin in daily energy flow. Frontiers in Neuroendocrinology 14:303-347.

$\rightarrow$ Garcia, V., P. Jouventin, and R. Mauget. 1996. Parental care and the prolactin secretion pattern in the king penguin: an endogenously timed mechanism? Hormones and Behaviour 30:259-265.

$\rightarrow$ Green, J. A., I. L. Boyd, A. J. Woakes, N. L. Warren, and P. J. Butler. 2005. Behavioural flexibility during year-round foraging in macaroni penguins. Marine Ecology Progress Series 296:183-196.

Hector, J. A. L., and A. R. Goldsmith. 1985. The role of prolactin during incubation: comparative studies of three Diomedea albatrosses. General and Comparative Endocrinology 60:236-243.

Holberton, R., B. Helmuth, and J. C. Wingfield. 1996. The corticosterone stress response in gentoo and king penguin during the non-fasting period. Condor 98:850-854.

Holberton, R. L. 1999. Changes in patterns of corticosterone secretion concurrent with migratory fattening in a Neotropical migratory bird. General and Comparative Endocrinology 116:49-58. 
$\rightarrow$ Landys, M. M., M. Ramenofsky, and J. C. Wingfield. 2006. Actions of glucocorticoids at a seasonal baseline as compared to stressrelated levels in the regulation of periodic life processes. General and Comparative Endocrinology 148:132-149.

$\rightarrow$ Lormeé, H., P. Jouventin, O. Chastel, and R. Mauget. 1999. Endocrinı correlates of parental care in an Antarctic breeding seabird, the emperor penguin, Aptenodytes forsteri. Hormones and Behaviour 35:9-17.

$\rightarrow$ Love, O. P., C. W. Breuner, F. Vézina, and T. D. Williams. 2004 Mediation of a corticosterone-induced reproductive conflict. Hormones and Behavior 46:59-65.

$\rightarrow$ McEwen, B. S., and J. C. Wingfield. 2003. The concept of allostasi in biology and biomedicine. Hormones and Behavior 43:2-15.

$\rightarrow$ Miller, D. A., C. M. Vleck, and D. L. Otis. 2009. Individual variation in baseline and stress-induced corticosterone and prolactin levels predicts parental effort by nesting mourning doves. Hormones and Behaviour 56:457-464.

$\rightarrow$ Newman, A. E. M., E. H. Chin, K. L. Schmidt, L. Bond, K. E. WynneEdwards, and K. K. Soma. 2008. Analysis of steroids in songbird plasma and brain by coupling solid phase extraction to radioimmunoassay. General and Comparative Endocrinology 155:503510.

Plikaytis, B. D., P. F. Holder, L. B. Pais, S. E. Maslanka, L. L. Gheesling, and G. M. Carlone. 1994. Determination of parallelism and nonparallelism in bioassay dilution curves. Journal of Clinical Micro biology 32:2441-2447.

$\rightarrow$ Pravosudov, V. V. 2003. Long-term moderate elevation of corticosterone facilitates avian food-caching behaviour and enhances spatial memory. Proceedings of the Royal Society B: Biological Sci ences 270:2599-2604.

$\rightarrow$ Ricklefs, R. E., and M. Wikelski. 2002. The physiology-life history nexus. Trends in Ecology \& Evolution 17:462-468.

$\rightarrow$ Romero, L. M. 2002. Seasonal changes in plasma glucocorticoid con centrations in free-living vertebrates. General and Comparative Endocrinology 128:1-24.

$\rightarrow$ Romero, L. M., J. M. Reed, and J. C. Wingfield. 2000. Effects of weather on corticosterone responses in wild free-living passerine birds. General and Comparative Endocrinology 118:113-122.

$\rightarrow$ Ropert-Coudert, Y., A. Kato, J. Baudat, C.A. Bost, Y. Le Maho, and Y. Naito. 2001. Feeding strategies of free-ranging Adélie penguins Pygoscelis adeliae analysed by multiple data recording. Polar $\mathrm{Bi}$ ology 24:460-466.

$\rightarrow$ Sakamoto, K. Q., K. Sato, M. Ishizuka, Y. Watanuki, A. Takahashi, F. Daunt, and S. Wanless. 2009. Can ethograms be automaticall, generated using body acceleration data from free-ranging birds? PLoS ONE 4:e5379.

$\rightarrow$ Sapolsky, R. M., L. M. Romero, and A. U. Munck. 2000. How to glucocorticoids influence stress responses? integrating permissive, suppressive, stimulatory, and preparative actions. Endocrine Reviews 21:55-89.
Schmidt, K. L., and K. K. Soma. 2008. Cortisol and corticosterone in the songbird immune and nervous systems: local versus systemic levels during development. American Journal of Physiology 295: R103-R110.

Silverin, B. 1986. Corticosterone-binding proteins and behavioural effects of high plasma levels of corticosterone during the breeding period. General and Comparative Endocrinology 64:67-74.

$\rightarrow$ Simeone, A., and R. P. Wilson. 2003. In-depth studies of Magellanic penguin (Spheniscus magellanicus) foraging: can we estimate prey consumption by perturbations in the dive profile? Marine Biology 143:825-831.

Sockman, K. W., P. J. Sharp, and H. Schwabl. 2006. Orchestration of avian reproductive effort: an integration of the ultimate and proximate basis of flexibility in clutch size, incubation behaviour, and yolk androgen. Biological Reviews 81:629-666.

Takahashi, A., M. J. Dunn, P. N. Trathan, J. P. Croxall, R. P. Wilson, K. Sato, and Y. Naito. 2004. Krill-feeding behaviour in a chinstrap penguin Pygoscelis antarctica compared with fish-eating in $\mathrm{Ma}$ gellanic penguins Spheniscus magellanicus: a pilot study. Marine Ornithology 32:47-54.

Vleck, C. M., L. L. Ross, D. Vleck, and T. L. Bucher. 2000. Prolactin and parental behaviour in Adélie penguins: effects of absence from nest, incubation length, and nest failure. Hormones and Behaviour 38:149-158.

$\rightarrow$ Walker, B. G., J. C. Wingfield, and P. D. Boersma. 2005. Age and food deprivation affects expression of the glucocorticosteroid stress response in Magellanic penguin (Spheniscus magellanicus) chicks. Physiological and Biochemical Zoology 78:78-89.

Walker, T. R., I. L. Boyd, D. J. McCafferty, N. Huin, R. I. Taylor, and K. Reid. 1998. Seasonal occurrence and diet of leopard seals ( $H y$ drurga leptonyx) at Bird Island, South Georgia. Antarctic Science 10:75-81.

Washburn, B. E., D. L. Morris, J. J. Millspaugh, J. Faaborg, and J. H. Schulz. 2002. Using a commercially available radioimmunoassay to quantify corticosterone in avian plasma. Condor 104:558563.

Williams, T. D. 1995. The penguins. Oxford University Press, Oxford. - 2008. Individual variation in endocrine systems: moving beyond the "tyranny of the Golden Mean." Philosophical Transactions of the Royal Society B: Biological Sciences 363:1687-1698.

$\rightarrow$ Williams, T. D., and J. P. Croxall. 1991. Annual variation in breeding biology of macaroni penguins, Eudyptes chrysolophus, at Bird Island, South Georgia. Journal of Zoology (London) 223:189-202.

Wingfield, J. C., C. W. Breuner, P. Honey, J. Jacobs, S. Lynn, D. Maney, M. Ramenofsky, and R. Richardson. 1998. Ecological bases of hormone-behavior interactions: the "emergency life history stage.” American Zoologist 38:191-206.

Associate Editor: Suzanne Henson Alonzo Editor: Ruth G. Shaw 